\title{
The Influence of "Smartwatch" Attributes towards Users' Purchase Intentions in the Bangkok Metropolitan Area
}

\section{Thananporn SETHJINDA* and Supawat KITWIMONTRAKUL}

\author{
Assumption University of Thailand, Hua Mak, Bang Kapi, Bangkok 10240, Thailand
}

('Corresponding author's e-mail: thananpornsth@au.edu)

Received: 5 March 2019, Revised: 22 September 2019, Accepted: 19 October 2019

\begin{abstract}
This study aims to develop understanding of the relationship between the product attributes of smartwatches, emotional and functional customers' experiences, and purchase intentions. The attributes examined in this study include Connectivity (CN), Interactivity (IA), Sense of Presence (SP), Intelligence (IT), Convenience (CV), and Security (S) among users of smartwatches in the Bangkok Metropolitan area. An on-line survey questionnaire was conducted among 400 respondents during July - October 2018. Multiple Regression was used to test the hypothesized relationship. Results showed that Sense of Presence (SP), Intelligence (IT), Convenience (CN), and Connectivity (CN) positively influenced emotional experience, whereas Intelligence (IT), Security (S), Sense of Presence (SP), Interactivity (IA), and Convenience $(\mathrm{CV})$ positively influenced functional experience. Both emotional experience and functional experience positively influenced users' purchase intentions $\left(R^{2} 32.8 \%\right)$. The results of this study support the importance of customers' experiences in smartwatch purchase decision making and so the key elements of product attributes that smartwatch developers should pay attention to are proposed.
\end{abstract}

Keywords: Smartwatch, Attributes of Smartwatch, Purchase Intention, Functional Experience, Emotional Experience

\section{Introduction}

With continual technological revolution, consumers around the world are witnessing consistent innovation, enabled by the Internet of Things to improve quality of life in multiple aspects. Daily objects, ranging from watches, eyeglasses, earpieces, wristbands, and home devices, have embedded intelligence and connectivity through the internet network [1], with functional features based around fitness, healthcare, travelling, and communication [2]. During the past few years, wearable devices have expanded globally at a growth rate of 10 and $13.2 \%$ during 2017 and 2018. Growth is predicted to continue at a compound annual rate of $13.4 \%$ during 2018 to 2022 . Within the wearable categories, smartwatches have exhibited stable growth and have commanded the highest average selling prices. In monetary term, the sales of smartwatches, measured by values, are estimated to account for two thirds of the wearable market. In terms of projected volume growth, sales of smartwatches from 2018 to 2022 are expected to grow by an average compounded growth rate of $17.9 \%$ per year [3].

Despite the increasing popularity of smartwatches and its promising projection, adoption has been much slower. According to eMarketer, smartwatch adoption had yet to become mainstream for consumers, when compared with the rate of adoption for smartphones or tablets as a percentage of internet users [4]. Researchers suggest that deeper levels of understanding of influencing factors of purchase intentions are needed to support wider adoption and product development [5]. In line with this observation, research interests in consumer adoption of smartwatches has accumulated during the past few years. Several researchers applied the framework of the Technology Acceptance Model (TAM) to examine the influence of perceived usefulness and perceived ease of use on users' attitudes and 
behavioral intentions towards smartwatches. Several drivers were added to the original TAM to account for possible cognitive and affective factors for adoption of smartwatches.

Specifically, Kim and Shin [5] proposed affective quality and relative advantage as the antecedent of perceived usefulness, while mobility and availability were proposed as predictors of perceived ease of use. Secondly, Chuah et al. [6] added visibility to the original model of TAM, and further examined the relationship between technology and fashion towards perceived usefulness and visibility [6]. Similarly, Choi and Kim [7] suggested that smartwatch technology attributes influenced perceived usefulness and perceived ease of use, while smartwatch luxury attributes influenced perceived enjoyment and perceived self-expressiveness. The importance of hedonic factors was further reflected in the work of Wu et al. [8] and Hsiao and Chen [9]. While the former proposed relative advantage, ease of use, compatibility, result demonstrability, enjoyment, and social influence as antecedents of attitudes and behavior intentions [8], the latter suggested that adoption of smartwatches should be examined from the perspectives of software, hardware, and aesthetic design [9].

With the increasing levels of competition, leading brands are continuously enabling a wide range of functionalities in smartwatches, such as health tracking, reminders, or even remotely-controlled paired devices at home. With this context, research on smartwatches should focus on answering the valueproposition of functionalities and applications of smartwatches [5]. Based on the existing studies, this study proposes to broaden smartwatch products' attributes and identify the mechanism through which such attributes could influence consumers' purchase intentions. This study proposes to adapt the IoT attributes discussed in the study of Chang et al. [10] to examine the relationship between smartwatch attributes with emotional experience, functional experience, and purchase intention. By looking at Connectivity, Interactivity, Presence, Intelligence, Convenience, and Security, this study broadens the attributes of smartwatches to address possible future areas of functionality enhancement [10]. At the same time, the study contributes alternative views on how such attributes are linked to customers' experiences and purchase intentions to academic literature.

\section{Literature review}

Smartwatches are defined as wrist-worn devices with computational power and with the ability to connect to other devices via short-range wireless connectivity, enabling them to send notifications, to collect and store personal data for health-related applications, and other possibilities [9,11] Smartwatches had their root in Seiko's Pulsar NL C01, the first digital watch with user-programmable memory [5]. With continual technological advancement that markedly reduced the size of electronic parts and enabled mass scale production, the Watchpad, a prototype of the modern form of smartwatch, arrived on the market in 2000, with a 32-bit ARM processor, 16 megabytes of memory, a fingerprint scanner, a speaker, and a microphone. During 2004 to 2012, various brands of smartwatch were introduced, fueling continual evolution of product design and functionality. Among the pioneers was Microsoft, which offered a shortlived subscription-based Smart Personal Object Technology, or SPOT, watch using radio connectivity. Other players, such as Samsung, Sony Ericsson, Motorola, and even Google, with multiple partnerships, offered a variety of models, featuring touchscreens, fitness information, email, and message notification functionalities [12]. When Apple's iWatch reached the market in 2015, it quickly achieved the leading market share, and spurred the penetration of smartwatches in multiple segments.

Which factors have been key to consumers' adoption of smartwatches? According to the existing empirical evidence, two key observations could be made. Firstly, several studies confirm the importance of perceived usefulness in influencing consumers' attitudes and behavioral intentions towards smartwatches [7]. Secondly, smartwatch adoption is not driven solely by technology benefits. Several studies found that customers' emotional values played a role in influencing consumers' attitudes, which directly influenced behavioral intentions. For instance, Kim and Shin [5] improved the explanatory power of TAM by conceptualizing the psychological quality of smartwatches as antecedents of consumers' affective factors. Similarly, Hsiao and Chen [9] found that the hardware, software, and aesthetic design of smartwatches directly influenced social value, performance value, emotional value, and value of money, all of which had a direct influence on intention to use smartwatches. 
To understand the relationship among attributes of smartwatches, customers' experiences, and purchase intentions, this study adapts the conceptual framework of Chang et al. [10]. The proposed model explores the influence of six product attributes towards customers' emotional and functional experiences and the relationship with purchase intentions. Each of the constructs are discussed below.

\section{Connectivity}

According to Chang et al. [10], connectivity refers to the degree to which objects are interconnected to facilitate communication among them. Objects may be connected through various channels and networks, creating opportunities for data collection and information analysis. Connectivity is regarded as an important feature of smartwatches. The earliest version of the smartwatch required pairing with mobile devices through Bluetooth. Recent reports showed that smartwatch producers were launching newer models with multiple connectivity, such as cellular, LTE, NFC, Wi-Fi, Bluetooth, or combinations of these [13]. Smartwatch connectivity was recognized as being flexible, bi-directional, and real-time, enabling a wide range of applications, especially in healthcare. To leverage smartwatch connectivity, Kheirkhahan et al. [14] explored the framework of using smartwatches and smartphones for real-time, online assessment and mobility monitoring. From the users' standpoints, connectivity allows customers to communicate anywhere and anytime. In the case that a smartwatch lacked stable connectivity, users would not experience the smoothness of the product, resulting in negative user experiences. Therefore, it is hypothesized that connectivity is positively related to consumers' functional and emotional experiences.

H1a: Connectivity has a positive influence on consumers' emotional experiences.

$\mathrm{H} 2 \mathrm{a}$ : Connectivity has a positive influence on consumers' functional experiences.

\section{Interactivity}

Communication technology has created an environment in which participants can communicate (oneto-one, one-to-many, and many-to-many), both synchronously and asynchronously, and participate in reciprocal message exchanges [15]. Extending this concept to technological interactivity, Kim [16] suggested that interactivity was narrowed to user-to-system interactivity in multidirectional perspectives. Specifically, technological interactivity covered three components, namely, user control, responsiveness, and synchronicity. Previous research found that smart devices might affect interactivity in the new media, as the users were able to control the timing, content, and sequence of communication, and judge the responsiveness of communication feedback, such as replies to emails or actions taken by the system based on feedback provided [16]. Smartwatches have been developed to incorporate multidirectional interactivity, ranging from communication, payment, tracking, and data storage of individual health related activities, with more possibilities currently being explored. Studies have found that interactivity improved the efficiency and usefulness of information and communication technology [17]. Hence, it is hypothesized that interactivity has a positive relationship with functional and emotional experiences.

$\mathrm{H} 1 \mathrm{~b}$ : Interactivity has a positive influence on consumers' emotional experiences.

$\mathrm{H} 2 \mathrm{~b}$ : Interactivity has a positive influence on consumers' functional experiences.

\section{Sense of presence}

According to Chang et al. [10], the concept for sense of presence was derived from the concept of telepresence. In the context of communication, they posited that telepresence could influence consumers' subjective feelings about products, based on media representation in physical and social environments. Consumers' feelings about the appearance of products on the scene promoted positive evaluation of the product, creating positive experiences for the consumers. With respect to IoT devices, Pinochet et al. [18] associated sense of presence to potential application in the prevention of disease and the management of public and environmental crises. The attribute that enables smartphones to remotely control other devices, such as starting air conditioners, is considered to be a sense of presence. As for smartwatches, the attribute of sense of presence is reflected in its feature allowing the location of lost or misplaced phones [19], or controlling channels, viewing programs, and recording shows on Sweden's provider of Tivo 
devices [20]. Hence, the third attribute of smartwatches relates to the sense of presence, which will in turn positively influence consumers' functional and emotional experiences [10]. The following hypotheses are proposed.

H1c: Sense of presence has a positive influence on consumers' emotional experiences.

$\mathrm{H} 2 \mathrm{c}$ : Sense of presence has a positive influence on consumers' functional experiences.

\section{Intelligence}

Ritter et al. [20] defined intelligence as tasks performed by an object with complicated recognition functions, data processing, judgement, and implementation. In terms of product features, intelligence refers to the degree to which functions are automated through built-in sensing, memory systems, data processing, reasoning, and communication capacity. Several intelligence features, such as notification of incoming messages and emails and fitness tracking, are present in current models of smartwatches on the market. Providers have been introducing artificial intelligence in smartwatches to enable "personal assistant" functions, such as nutritional advice, restaurant recommendations, and goal tracking [19]. Smartwatch intelligence has been explored by researchers for potential healthcare applications, such as automatic fall-detection systems [21], self-management of pediatric asthma [22], and combined smartphone and smartwatch diabetes diary application [23]. Although the research remains at an early stage, results have shown user satisfaction and improvement in preventive care [22]. With the speed of innovation and technological advancement, the following hypotheses are proposed.

H1d: Intelligence has a positive influence on consumers' emotional experiences.

$\mathrm{H} 2 \mathrm{~d}$ : Intelligence has a positive influence on consumers' functional experiences.

\section{Convenience}

Convenience is associated with the degree to which consumers could save their time and effort in considering, purchasing, and using products [24]. In the context of this study, convenience refers to consumers' perceptions of smartwatches in providing increasing efficiency in users' activities [10]. Ogbanufe and Gerhart [25] found that convenience characteristics of smartwatches were one of the key factors, directly affecting the confirmation expectation of smartwatches and indirectly affecting users' satisfaction [25]. An example of smartwatch features reflecting convenience is navigation. Some models can send different vibration patterns, indicating whether users should turn left or right, when following directions. Moreover, users can remain connected on smartwatches longer than on smartphones, given longer battery time and the ability to wear smartwatches in such circumstances as swimming. In fact, a users' survey revealed that users purchased smartwatches in addition to smartphones because they preferred the ease of handy access to the device [26]. In short, it is hypothesized that convenience positively influences functional experience and emotional experience.

H1e: Convenience has a positive influence on consumers' emotional experiences.

H2e: Convenience has a positive influence on consumers' functional experiences.

\section{Security}

Based on the work of Chang et al. [10], consumers' perceptions of the level of security with respect to a provider or a product is an important factor for purchase decisions. With the continual rise in connected devices, researchers in IT security were concerned about possible exploitation by cyber criminals. With respect to smartwatches, Liu et al. investigated the possible exploitation of accelerometers, which were embedded in smartwatches to track users' hand movements. They studied possible side-channel attack to infer user inputs on keyboards. Although there has not been any report of security breaches of smartwatches, the increasing potential application demands high levels of standards for data security and privacy [27]. Security consideration is, therefore, an important attribute of smartwatch purchase consideration. In accordance with this observation, it is hypothesized that:

H1f: Security has a positive influence on consumers' emotional experiences.

H2f: Security has a positive influence on consumers' functional experiences. 


\section{Functional and emotional experiences and purchase intentions}

Consumers' experiences are one of the most important factors in the purchase-decision making process [18]. In fact, experience has been investigated in several aspects, such as in consumer research, product design and engineering, and product usage [28]. More specifically, Hekkert [29] defined product experience as "the entire set of affects that is elicited by the interaction between a user and a product, including the degree to which all our senses are gratified (aesthetic experience), the meanings we attach to the product (experience of meaning) and the feelings and emotions that are elicited (emotional experience)" (p. 160).

With regards to technology-related devices, functional experience refers to the degree of consumers' satisfaction with the perceived usefulness and perceived quality of the technology in product and services. Functional experience could be determined by the expectation of the services and products to be offered to them, as well as the post-usage perception of them [11]. On the other hand, emotional experience relates to consumers' feelings that have developed beyond reasons or usage of products. These emotional experiences may encompass the feelings of trust, esteem, closeness, joy, or frustration developed in the process of consumers' interactions with the products [18]. Both functional and emotional experience could have an impact on consumers' purchase intentions. According to Yang [30], consumers' evaluations and feelings from experience could influence their attitudes and future purchase intentions, as well as the likelihood of recommending products or services to others [30]. Therefore, this study proposes that functional and emotional experiences could exert positive influence on consumers' intentions $[11,18]$. The following hypotheses are proposed.

H3a: Emotional experience has a positive influence on purchase intentions of smartwatches.

H3b: Functional experience has a positive influence on purchase intentions of smartwatches.

\section{Conceptual framework}

In line with the framework of Pinochet et al. [18] and Chang et al. [10], the conceptual framework is demonstrated below (Figure 1):

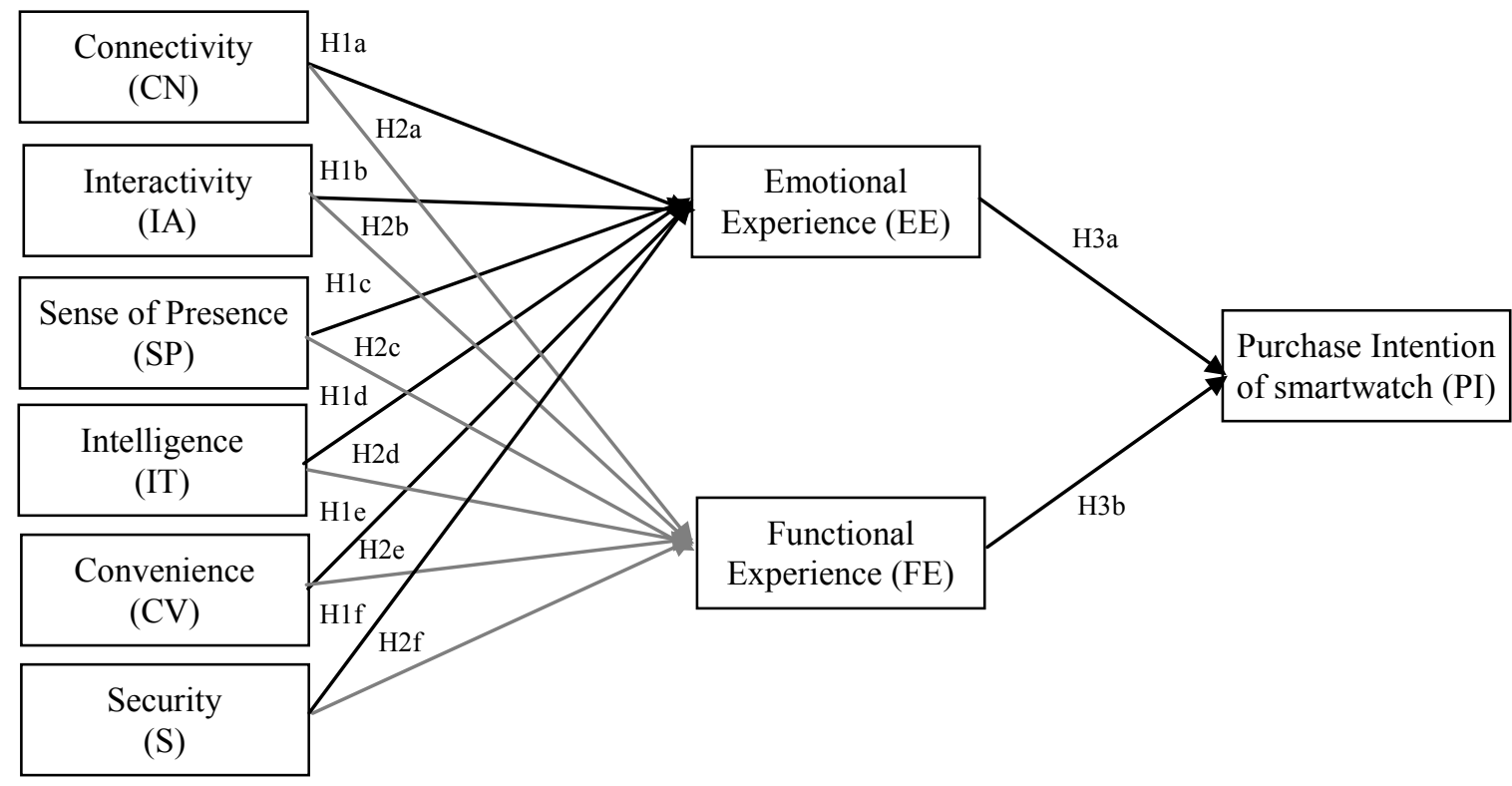

Figure 1 Conceptual Framework. 


\section{Materials and methods}

Data collection and analysis

The respondents for this study were residents of Bangkok who had prior experience with smartwatches and were considering the purchase of a new one. The questions were developed from the model of Chang et al. [10], using a Five-point Likert scale as a measurement. Data collection was performed through an online survey questionnaire by convenience sampling through the researchers' network of friends, from bachelor to master level, as well as colleagues from professional circles. Respondents were also asked to forward the questions to their friends who currently used smartwatches and were considered buying a new one. The pre-test was conducted among 32 respondents to confirm the reliability and validity of the instrument. The results of reliability test is shown in Table 1. Upon completion, the survey was administered during July to October 2018. The questionnaires reached 445 respondents. After validation, only 400 responses were qualified for the study. Multiple regression was used to test the hypothesized relationships.

Table 1 Cronbach's Alpha and mean of the variables.

\begin{tabular}{cccc}
\hline Variables & Cronbach's Alpha & Number of Items & Mean \\
\hline Connectivity & 0.846 & 3 & 4.24 \\
Interactivity & 0.911 & 3 & 4.13 \\
Sense of Presence & 0.927 & 3 & 3.85 \\
Intelligence & 0.946 & 3 & 4.15 \\
Convenience & 0.956 & 3 & 4.08 \\
Security & 0.816 & 3 & 3.71 \\
Emotional Experience & 0.963 & 5 & 3.73 \\
Functional Experience & 0.934 & 5 & 3.96 \\
Purchase Intention & 0.868 & 4 & 4.17 \\
\hline
\end{tabular}

\section{Results and discussion}

\section{Demographic profile summary}

Table 2 reports the demographics of 400 respondents. Overall, the respondents were in the Millennial and Gen Y age group, primarily aged between 20 - 29 years old, followed by 30 - 39 years old, representing $80 \%$ of the respondents (Table 2). In terms of gender, female respondents accounted for 58 $\%$, with the remaining $42 \%$ were male. For education level, the respondents graduated with Bachelor degree (65\%), followed by Master degree (29\%). Monthly incomes were in the range of THB 20,001 45,000 or USD $650.00-1,500$ (41\%), and THB 45,001 - THB 70,000 or USD 1,501 - 2,300 (28\%) (Table 2). The sample profiles closely reflect the users of smartwatches in Thailand, as reported in a survey of smartwatch users in Thailand in 2017. According to the report, female users accounted for a relatively higher percentage, compared to males. In terms of age, the main users were in the age range of 25 - 34 years old, accounting for $40.6 \%$, followed by 35 - 44 years old (23.6\%) and 18 - 24 years old $(18.9 \%)[31]$. 
Table 2 Demographic profile summary of the sample.

\begin{tabular}{clcc}
\hline \multicolumn{2}{c}{ Demographical Data $(\mathbf{N}=\mathbf{4 0 0})$} & $\mathbf{N}$ & Frequency (\%) \\
\hline \multirow{2}{*}{ Gender } & Female & 233 & $58 \%$ \\
& Male & 167 & $42 \%$ \\
\hline \multirow{5}{*}{ Age } & Less than 20 years old & 10 & $2 \%$ \\
& $20-29$ years old & 253 & $63 \%$ \\
& $30-39$ years old & 108 & $27 \%$ \\
& $40-49$ years old & 15 & $4 \%$ \\
& $50-59$ years old & 14 & $4 \%$ \\
& 60 years old or above & 0 & $0 \%$ \\
\hline \multirow{3}{*}{ Education } & $<$ Bachelor degree & 25 & $6 \%$ \\
& Bachelor degree & 258 & $65 \%$ \\
& Master degree & 117 & $29 \%$ \\
\hline \multirow{5}{*}{ Monthly Income } & Below 20,000 Baht & 36 & $9 \%$ \\
& $20,001-45,000$ Baht & 163 & $40 \%$ \\
& $45,001-70,000$ Baht & 110 & $28 \%$ \\
& $70,001-100,000$ Baht & 50 & $13 \%$ \\
& $100,001-150,000$ Baht & 28 & $7 \%$ \\
& More than 150,000 Baht & 13 & $3 \%$ \\
\hline
\end{tabular}

\section{Pearson's correlation}

All variables in the study demonstrate positive correlations among one another, confirming the hypothesized relationship among the research variables. Tables 3 - 5 show that the Pearson's Correlation Matrix for $\mathrm{H} 1 \mathrm{a}-\mathrm{H} 1 \mathrm{f}, \mathrm{H} 2 \mathrm{a}-\mathrm{H} 2 \mathrm{f}$ and $\mathrm{H} 3 \mathrm{a}-\mathrm{H} 3 \mathrm{~b}$ are positively correlated with $\mathrm{P}$-values at less than 0.05 . A high level of correlations is observed among Connectivity $(\mathrm{CN})$ and Interactivity (IA), positively correlated at 0.643 , and Intelligence (IT) and Convenience (CV), positively correlated at 0.622 (Table 3). Intelligence (IT) was positively correlated with Functional Experience (FE), correlated at 0.664, while Connectivity $(\mathrm{CN})$ and Interactivity (IA) were positively correlated at 0.643 (Table 4).

Table 3 Pearson's Correlation Matrix (H1a - H1f)

\begin{tabular}{llr|r|r|r|r|r|r} 
& \multicolumn{1}{c}{ EE } & \multicolumn{1}{c}{ CN } & IA & SP & IT & CV & S \\
\hline Pearson & EE & 1.000 & & & & & & \\
\cline { 2 - 10 } Correlation & CN & .290 & 1.000 & & & & & \\
\cline { 2 - 10 } & IA & .210 & .643 & 1.000 & & & & \\
\hline & SP & .553 & .328 & .539 & 1.000 & & & \\
\hline & IT & .520 & .423 & .431 & .535 & 1.000 & & \\
\hline & CV & .600 & .330 & .280 & .518 & .622 & 1.000 & \\
\hline & S & .411 & .307 & .260 & .327 & .545 & .485 & 1.000
\end{tabular}


Table 4 Pearson's Correlation Matrix (H2a-H2f).

\begin{tabular}{|c|c|c|c|c|c|c|c|c|}
\hline & & $\mathrm{FE}$ & $\mathrm{CN}$ & IA & SP & IT & $\mathrm{CV}$ & $\mathrm{S}$ \\
\hline Pearson & $\mathrm{FE}$ & 1.000 & & & & & & \\
\hline \multirow[t]{6}{*}{ Correlation } & $\mathrm{CN}$ & .321 & 1.000 & & & & & \\
\hline & IA & .273 & .643 & 1.000 & & & & \\
\hline & SP & .518 & .328 & .539 & 1.000 & & & \\
\hline & IT & .664 & .423 & .431 & .535 & 1.000 & & \\
\hline & $\mathrm{CV}$ & .570 & .330 & .280 & .518 & .622 & 1.000 & \\
\hline & $\mathrm{S}$ & .576 & .307 & .260 & .327 & .545 & .485 & 1.000 \\
\hline
\end{tabular}

Table 5 Pearson's Correlation Matrix (H3a- H3b).

\begin{tabular}{llr|r|r} 
& & \multicolumn{1}{c}{ PI } & \multicolumn{1}{c}{ EE } & \multicolumn{1}{l}{ FE } \\
\hline Pearson & PI & 1.000 & & \\
\cline { 2 - 5 } Correlation & EE & .398 & 1.000 & \\
\cline { 2 - 5 } & FE & .366 & $\mathbf{. 6 3 8}$ & 1.000
\end{tabular}

\section{Multiple regression analysis}

Results of multiple regression analysis are shown in Figure 2. For H1, results showed that Connectivity (H1a), Sense of Presence (H1c), Intelligence (H1d), and Convenience (H1e) had a statistically significant relationship with emotional experience, with $\mathrm{R}^{2}$ of 48.7 at $95 \%$ confidence level. The impact of Connectivity (H1a) and Security (H1f) on emotional experience were not supported. Among all the attributes, Sense of Presence had the highest degree of impact on emotional experience $(\beta$ $\left.=0.393,{ }^{*} p<0.01\right)$, followed by Convenience $\left(\beta=0.299,{ }^{* *} p<0.05\right)$, Intelligence $\left(\beta=0.122,{ }^{*} p<\right.$ $0.05)$, and Connectivity $(\beta=0.148, * p<0.01)$ (Figure 2$)$. The variance inflation factors (VIFs) confirmed that there was no issue on the multicollinearity, as the VIFs of all hypotheses were less than 0.05 .

In terms of functional experience, regression results showed that Interactivity ( $\mathrm{H} 2 \mathrm{~b})$, Sense of Presence (H2c), Intelligence (H2d), Convenience (H2d), and Security (H2f) had a positive impact on functional experience, with $\mathrm{R}^{2}$ of 56.1 at $95 \%$ confidence level. There was no statistical relationship between Connectivity $(\mathrm{H} 2 \mathrm{a})$ and Functional Experience. Among all smartwatch attributes, Intelligence had the strongest degree of influence on functional experience $\left(\beta=0.356,{ }^{*} p<0.01\right)$, followed by Security $\left(\beta=0.266,{ }^{*} p<0.01\right)$, Sense of Presence $\left(\beta=0.245,{ }^{*} p<0.01\right)$, Interactivity $\left(\beta=0.161,{ }^{*} p<\right.$ $0.01)$, and Convenience $(\beta=0.113, * * p<0.05)$ (Figure 2). Validation of multicollinearity is confirmed, as the VIFs of all hypotheses were less than 0.05 . 


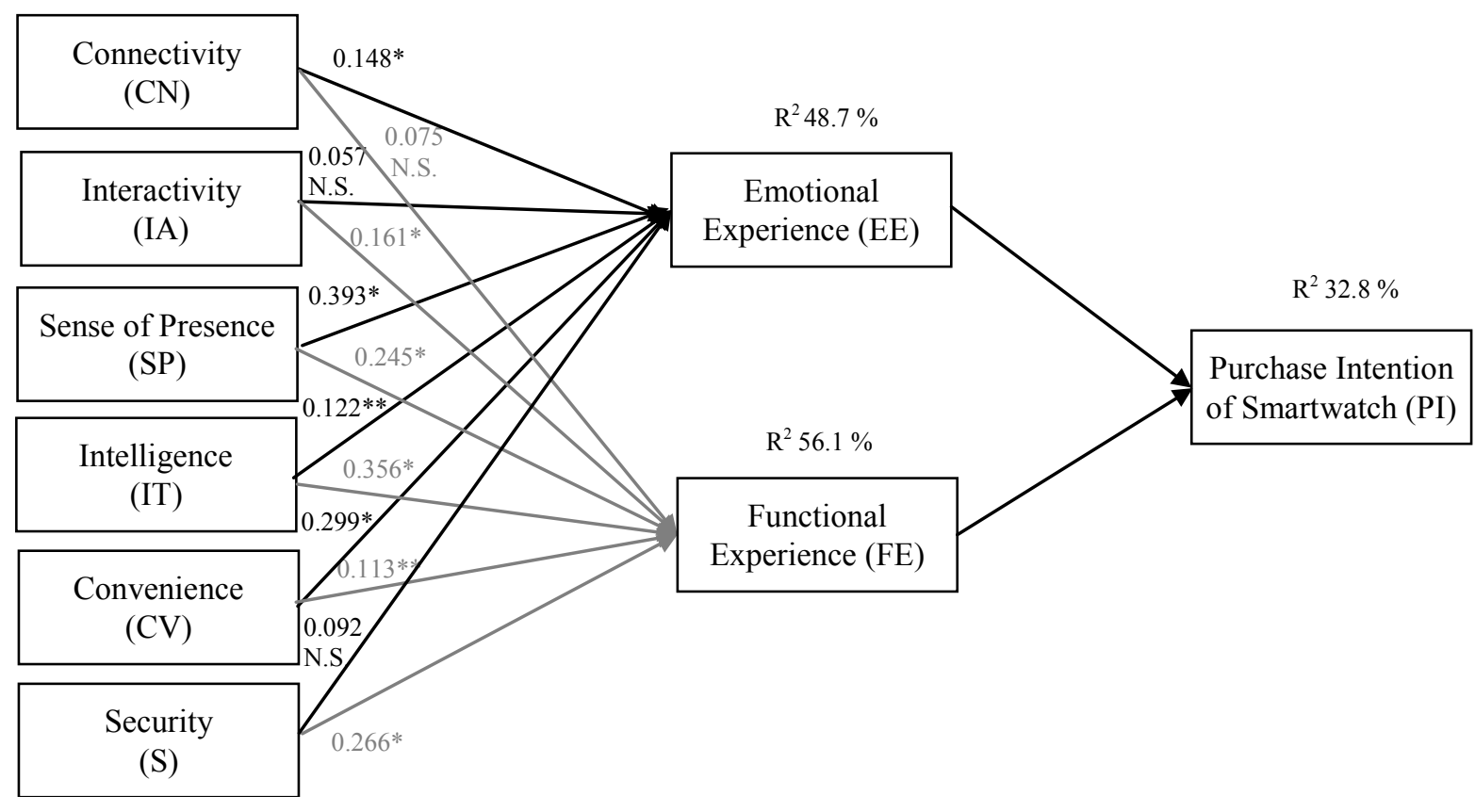

Note: significance $* p<0.01 ; * * p<0.05 ;$ N.S. $=$ Not Significant

Figure 2 Summary of statistical results.

\section{Discussion}

The preceding statistical results highlighted the following observations. Firstly, the impact of emotional experience was relatively stronger than that of functional experience. This finding renders support to the existing literature, suggesting that adoption of smartwatches was not driven purely by functional usefulness alone. Several researchers found that users regarded smartwatches as fashion [6], personalized and trendy items, reflecting individual identifies, emotions, and aesthetic values [5]. Studies of the Technology Acceptance Model of smartwatches found that affective quality had direct impact on perceived usefulness and users' attitudes towards smartwatches among target respondents in Korea [5]. In Taiwan, Hsiao and Chen [9] found that design aesthetics had the most significant effect on users' attitudes towards smartwatch adoption. The emphasis on design, along with functional values, was also one of the recommendations in the study of $\mathrm{Wu}$ et al. [8] and Adapa [32], suggesting that emotional factors were likely to play a part in smartwatch purchase decisions.

Secondly, although the impact of emotional experience was relatively stronger than that of functional experience, features and the functionality development of smartwatches could not be overlooked. All six attributes of smartwatches in this study played different roles in influencing users' purchase intentions. Specifically, Interactivity and Security, despite the lack of statistical significance on emotional experience, had significant impact on users' functional experiences. By the same token, connectivity, despite having its impact on emotional experience, does not have any impact on functional experience, as users place more emphasis on other attributes of smartwatches such as Intelligence, Security, Sense of Presence, Interactivity, and Convenience.

With regards to the second observation, the existing literature suggests multiple influencing factors at play with respect to the adoption of smartwatches. For instance, Bodin et al. [26] reported that the main reason that users purchased smartwatches was the expected convenience. Specifically, users did not want to continuously monitor devices or unlock devices for a quick view of information, and they liked the ease of handy access to smartwatches. Adapa [32] studied adoption of smartwatches using a qualitative 
approach. Her findings revealed that users considered the purchase of smartwatches based on fitness function and design. Hsiao and Chen [9] and Hsiao [33] suggested that smartwatch attributes of hardware, software, and design had significant direct effects on users' attitudes towards smartwatches. Similarly, Wu et al. [8] found that, despite the significance of aesthetic design, the relative advantage or features and functionalities of smartwatches had influenced users' attitudes and purchase intentions.

With respect to the results of this study, emotional and functional experiences of smartwatches should receive attention when planning marketing and product development. To support emotional experience, marketing of smartwatches might focus on demonstrating a sense of presence or the ability to perform various tasks remotely, and convenience showing how smartwatches could make their lives simpler. At the same time, functional experience of smartwatch might be promoted through the showcase of smartwatch intelligence. Other features, such as Security, Sense of Presence, and Interactivity, would most likely be valued by potential users of smartwatches, and should be included in product highlights.

In terms of functionality development, our findings suggest that smartwatches should continue to focus on developing advanced features, focusing on Intelligence, Sense of Presence, Security, Interactivity, and Convenience. At present, several providers have started to incorporate artificial intelligence and machine learning in order to provide recommendations based on data available through smartwatches. The future generation of smartwatches might incorporate advanced sensors and recommendations beyond the applications imaginable today.

\section{Conclusions}

To contribute to academic research on the adoption of smartwatches, this research explored how six attributes of smartwatches influenced emotional and functional experiences and purchase intentions. Based on a survey of 400 respondents in Bangkok, multiple regression showed that Connectivity, Sense of Presence, Intelligence, and Convenience had significant positive impacts on emotional experience, while Interactivity, Sense of Presence, Intelligence, Convenience, and Security had significant impacts on functional experience. It is important to note that Sense of Presence had the strongest influence on emotional experience, while Intelligence had the strongest influence on functional experience. Both emotional and functional experiences had significant positive relationships with purchase intentions of smartwatches among the target respondents. Emotional experience had a higher degree of impact on purchase intentions when compared with functional experience.

The results of this study showed that attributes of smartwatches played different roles in influencing users' purchase intentions. Users tended to associate features that allowed them to remotely control other devices, or Sense of Presence and Convenience with emotional experience, while Intelligence, Sense of Presence, and Security were the top three influencing factors of functional experience. Smartwatch developers could focus on these attributes when planning marketing and product development strategies. These attributes could also be expanded to other smart wearable devices.

The following limitations should be noted. Firstly, the results of the study could not be generalized to more diverse populations, given that the survey was conducted among residents of Bangkok. Future studies might conduct cross-comparison between residents in various areas within Thailand. Secondly, the study did not evaluate the effect of age or gender and whether there might be any differences on the influencing factors. Thirdly, although our findings added new dimensions to the attributes of smartwatches, namely security and sense of presence, the study did not take the aesthetic design or pricing range of smartwatches into consideration.

Future research should consider analysis of consumers' behaviors by different segmentation, such as the mass market or premium markets, to understand the similarities and differences among key factors, which may have an impact on users' adoption between these two segments. 


\section{Acknowledgements}

The authors wish to thank Dr. Rawin Vongurai, Program Director of Ph.D. in Innovative Technology Management, for his review of the manuscript and suggestions throughout the process.

\section{References}

[1] F Xia, LT Yang, L Wang and A Vinel. Internet of things. Int. J. Commun. Syst. 2012; 25, 1101-2.

[2] Forbes. Available at: www.forbes.com, accessed November 2018.

[3] IDC Worldwide Quarterly Wearables Tracker, Available at: https://www.idc.com, accessed October 2018.

[4] eMarketer, Available at emarketer.com, accessed September 2019.

[5] KJ Kim and DH Shin. An acceptance model for smartwatches: Implication for the adoption of future wearable technology. Internet Res. 2015, 25, 527-541.

[6] WSH Chuah, PA Rauschnabel, N Krey, B Nguyen, T Ramayah and S Lade. Wearable Technologies: The role of usefulness and visibility in smartwatch adoption. Comput. Hum. Behav. $2016 ; \mathbf{6 5}, 276-84$

[7] J Choi and S Kim. Is the smartwatch an IT product or a fashion product? A study on factors affecting the intention to use smartwatches. Comput. Hum. Behav. 2016; 63, 777-86.

[8] $\mathrm{LH} \mathrm{Wu}, \mathrm{LC} \mathrm{Wu}$ and SC Chang. Exploring consumers' intention to accept smartwatch. Comput. Hum. Behav. 2016; 64, 383-92.

[9] KL Hsiao and CC Chen. What drives smartwatch purchase intention? Perspectives from hardware, software, design and value. Telemat. Inform. 2018; 35, 103-13.

[10] Y Chang, D Xuebing and S Wei. Influence of characteristics of the internet of things on consumer purchase intention. Soc. Behav. Personal. 2014; 42, 321-30.

[11] TechTarget: IoT Agenda, Available at https://internetofthingsagenda.techtarget.com, accessed October 2018.

[12] TechRadar, Available at https://www.techradar.com, accessed October 2018.

[13] Dow Jones International News, Available at https://search.proquest.com/docview/1886189021? accountid=8401, accessed October 2018.

[14] M Kheirkhahan, S Nair, A Davoudi, P Rashidi, AA Wanigatuna, DB Corbett, T Mendoza, TM Manini and S Ranka. A smartwatch-based framework for real-time and online assessment and mobility monitoring. J. Biomed. Inform. 2019; 89, 29-40.

[15] S Kiousis. Interactivity: Concept explication. New Media Soc. 2002, 4, 355-83.

[16] KJ Kim. Interacting Socially with the Internet of Things (IoT): Effect of source attribution and specialization in Human-IoT interaction. J. Comput. Mediat. Comm. 2016; 21, 420-35.

[17] E Park, KJ Kim and SJ Kwon. Understanding the emergence of wearable devices as next-generation tools for health communication. Inform. Tech. People 2016; 29, 717-32.

[18] LHC Pinochet, EL Lopes, CHF Srulzon and LM Onusic. The influence of the attributes of "Internet of Things" products on functional and emotional experiences of purchase intention. Innovat. Manag. Rev. 2018; 15, 303-20.

[19] PR Newswire US, Available at https://prnews.com, accessed October 2018.

[20] Engadget, Available at engadget.com, accessed October 2018.

[21] E Casilari and MAO Jimenez. Automatic fall detection system based on the combined use of a smartphone and a smartwatch. PloS One 2015; 10, e0140929.

[22] A Hosseini, CM Buonocore, S Hashemzadeh, H Hojaiji, H Kalantarian, C Sideris, AAT Bui, CE King and M Sarrafzadeh. Feasibility of a secure wireless sensing smartwatch application for the self-management of pediatric asthma. Sensors 2017; 17, 1780.

[23] E Årsand, M Muzny, M Bradway, J Muzik and G Hartvigsen. Performance of the first combined smartwatch and smartphone diabetes diary application study. J. Diabetes. Sci. Technol. 2015; 9, 556-63. 
[24] M Brown, N Pope and K Vogues. Buying or browsing? An exploration of shopping orientations and online purchase intention. Eur. J. Mark. 2003; 37, 1666-84.

[25] O Ogbanufe and N Gerhart. Watch it! Factors driving continued feature use of the smartwatch. Int. J. Hum. Comput. Int. 2018; 34, 999-1014.

[26] WK Bodin, D Jaramillo, SK Marimekala and M Ganis. Security challenges and data implications by using smartwatch devices in the enterprise. In: Proceedings of the $12^{\text {th }}$ International Conference \& Expo on Emerging Technologies for a Smarter World. Melville, USA, 2015.

[27] SR Peppet. Regulating the internet of things: First steps toward managing discrimination, privacy, security, and consent. Texas Law Rev. 2014, 93, 85-178.

[28] P Desmet and P Hekkert. Framework of product experience. Int. J. Des. 2007, 1, 57-66.

[29] P Hekkert. Design aesthetics: Principles of pleasure in product design. Psychol. Sci. 2006, 48, $157-$ 72.

[30] SL Yang. 2004, Environmental Experience, Experience Value, Customer Satisfaction and Intention. Ph.D. Dissertation, University of Science and Technology, Taipei, Taiwan.

[31] Statista, Available at https://statista.com, accessed September 2019.

[32] A Adapa. 2016, Factors Influencing the Adoption of Smart Wearable Devices. Master Theses, Missouri University of Science and Technology, Missouri, USA.

[33] K Hsiao. What drives smartwatch adoption intention? Comparing Apple and non-Apple watch. Libr. Hi Tech. 2017; 35, 186-206. 